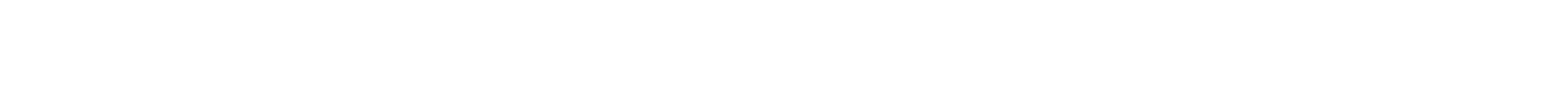 ورلمة خواصها البصربة
}

\author{
جلا إبرالهيم جلمم

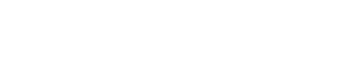 \\ ك ـ لية الـ علوم \\ جلمعة الموصل
}

(تاريخ الإتلام 17/ 6 /2012 ؛ تاريخ القبول 10 /9 / 2012)

\section{الملغص}

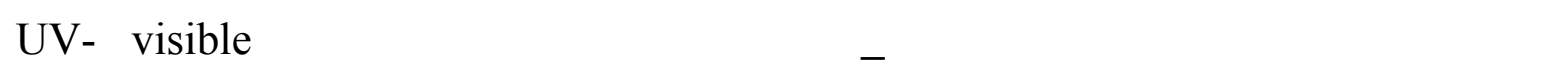
spectrophotometer بولنطة الأشعة للسينية Polyvinyl chloride) PVC)، إذ مق تشعيعه بجرع مقاوتة من الأشعة فوق

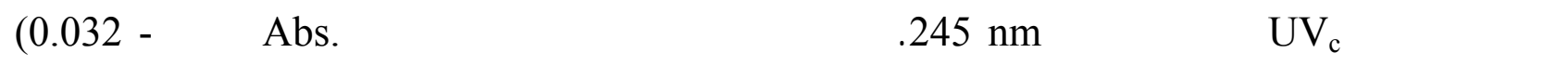

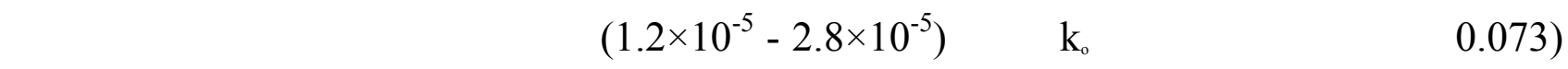

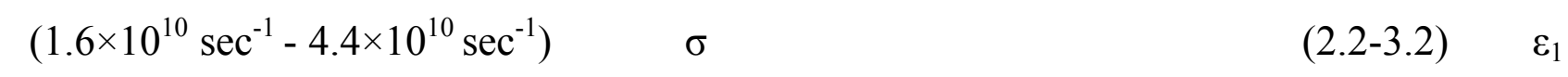
لأق لـ جرء بة بشعاعية (102859 erg/mm² النتائج أن هنك علاقة طرعية ه ـ ـ ـ ابين الخواص البصرية أعلاه وجرعة الأثعة فوق البفسجية. التضح أن هنك إمكانية ء الية للمست خدلم ال فلم اللشعاعي مجراعاً للأشعة فوق البن فسجية.

الكاملت الدالة: أفلام التشخيص الفوتوغرافي، مجراع، الخواص البصرية، الأشعة ف ـوق البف سجية UV. 
حلا إبراهيم جلنم

\title{
The Possibility of Using Photographic Diagnostic Films as Dosimeter of UV-Radiation and Study the Optical Properties of these Films
}

\author{
Hala I. Gasem \\ Department of Physics \\ College of Science \\ University of Mosul
}

\begin{abstract}
UV- visible spectrophotometer was used to determine the optical properties of the dental x-ray radiation films poly (vinyl chloride). The films were irradiated with varying doses of ultraviolet radiation $\mathrm{UV}_{\mathrm{c}}$ with $245 \mathrm{~nm}$. Optical absorbance peaks Abs. ranged from (0.032- 0.073$)$, the peaks of extinction coefficient $\mathrm{k}_{\mathrm{o}}$ ranged from $\left(1.2 \times 10^{-5}-2.8 \times 10^{-5}\right)$, while the peaks of the real dielectric constant $\varepsilon_{1}$ were found to be between the (2.2-3.2), and the of the optical conductivity $\sigma$ ranged from $\left(1.6 \times 10^{10} \mathrm{sec}^{-1}-4.4 \times 10^{10} \mathrm{sec}^{-1}\right)$. The above data were obtained for lower radiation dose $\left(102859 \mathrm{erg} / \mathrm{mm}^{2}\right)$ and higher radiation dose $(720014$ $\mathrm{erg} / \mathrm{mm}^{2}$ ). The results showed that there is a direct correlation between the optical properties and radiation dose. The results revealed that there is a high possibility for using the dental $\mathrm{X}$-ray radiation films as dosimeters of $\mathrm{UV}_{\mathrm{c}^{-}}$-radiation.
\end{abstract}

Keywords: Dental x-ray radiation film, dosimeter, optical properties, $\mathrm{UV}_{\mathrm{c}}$-radiation.

\section{|لإمة}

يتكون الفلم الفوتوغرافي Poly(vinyl chloride) PVC ذو الص يغة الك ـيميائية (Fluka, 2007/2008) والمستخم لأغراض التشخيص للثعاعي بولسطة اللثعة للسينية من صفيحة رقيقة من ماةشففةة إما من مادة للسليلوز (Cellulose) أو من مادة البولستر (Polyester) مضلفا إليه صبغة ذات لون ازرق (Stuart and Michael, 2000). يستخه الفلم الف ـوتوغرافي في مج الات عدية منها مجالات التشخيص في ط ـ ـ ـ ـ ـب الأسنان، ك ما ت مم اعتبارو مجراعا للأشعة للد ينية (Meredith and Massy, 1977) وذلك لما يحتويه من مواصفلت ومميزك مهمة كونه رخيص الثمن ومتوفرأ ولا يحتاج عند لستخدلمه إله طلة كهربائية ويسطبع

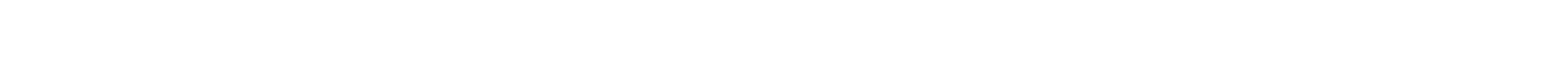
المسلسية.

لستخلمت مطيافية الضوء المرئي وفوق البفسجية في كثير من الدرلست التي تتناول التغيرات الحاصلة على المركبت الكيميائية والنماذج البيولوجية من خلل التغير الحاصل في الكثلفة البصرية عند الطول الموجي لتلك المركبت والنماذج، إذ لستخدت في معرفة التغيرك الحاصلة في تركيب البوليمرك أو 
(Akber et al., 1980) (Akivastara and Virk, 2000) (المواد بشكل عل نتيجة تسخينها أو تشعيعها (Akb) (بليمل، Tanu Sharma et al., 2007) فضلا عن لستخدلمها في إيجاد معلمل الامتصاص وفي قيلس التأثير للثعاعي للأثعة المؤينة وإيجاد الجرعة للثعاعية لبعض المواد المتعسة للشعاع من خلل التغير الطيفي الحاصل لتك المواد (محيميد و مسن، 1999 ) (Singh and Prasher, 2004) .

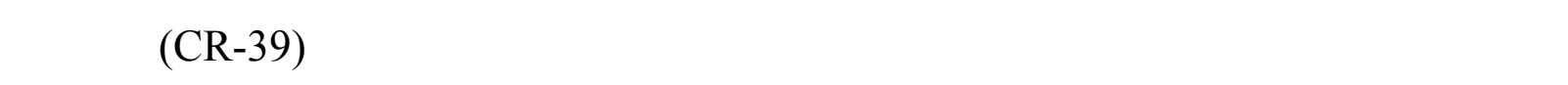

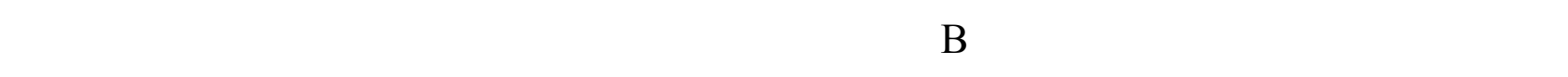
الأشخاص بشكل علم (Wong et al., 1992) (Shweikani et al., 2002)، كما لستعل ( الجبوري، 1984) طريقة كيميائية لتجريع الأثعة فوق البنهجية. توصل (Nagpal, 2000) اله لن معلملة المواد حراريا (CaF2:Eu, CaF2:Tb) تمتك هسلسية عالية جدا للمثشعة فوق البفسجية بطول 250nm وبهذا تستخه هذه المواد في قيلس جرعة الأشعة فوق البفسجية. لجريت درلسة من قل مل من (Nadeem and Ahmed, 2000) وأفلام (PhHgS) على التوالم، حيث مُ جسلب المتصاصية الضوئية A الانكسار n ممعلمل الخمود k مالتوصيلية البصرية الضوئية م وثابت العزل الكهربائي ع. تناولت الدرلسة التي قل بها (Deshmukh et al., 2008) تشعبع أفلام (PVC-PMMA) بالأشعة فوق البفسجية ودرلسة الخواص البصرية لتلك الأفلام من ضنمنها معلمل الامتصاص م والفانية البصرية

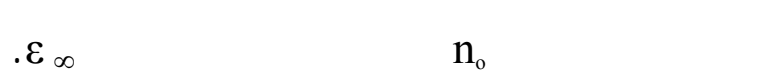
لما في البح ـث الحالي فستم درلس ـة إمكانية لستخدام الفلم الفوتوغرافي مجراعاَ للأثث ـ ـ ـ ع عة فوق البفسجية (UV-Visible light) من نوع UV ذالت الطلة العالية بلستخداd فقنية مطيفية الضوء المرئي وفوق البفسجية (UV-Visible Spectrophotometer) ودرلسة الخواص البصرية للفلم الفوتوغرافي بعد تشعيعه بالأثعة فوق البنفسجية.

\section{الجانب القالري}

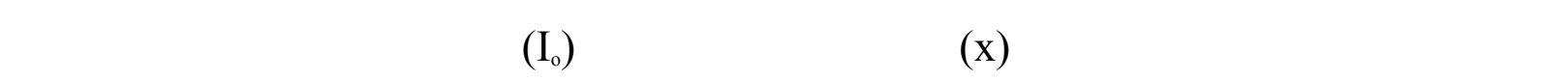
(I) بسب المتصاص والتشتت دلخل المادة والانعكلس منسطحه. إن السبب الرئيسي في نقليل للثدة هو

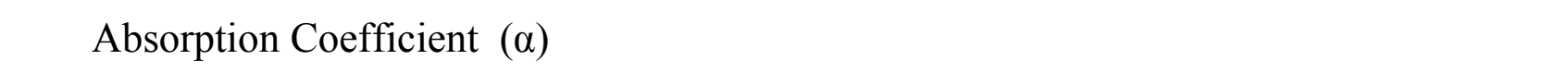
يعتمد علىطاقة الفوتون للسأط وعلى خصائص الماة ويعرف على انه قيلس لنسة الخسارة في الثعاع من

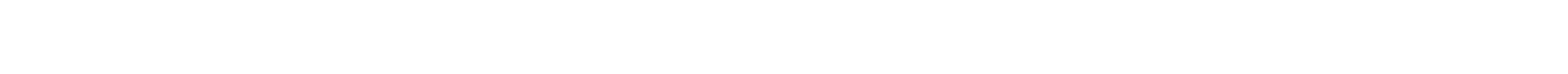
الانب - علث والامتصاص (Nadeem and Ahmed, 2000). $\mathrm{I}=\mathrm{I}_{\mathrm{a}} \mathrm{e}^{-\infty x}$ 
ومعادلة الامتصاصية A هي:

$A=\log \frac{I_{\bullet}}{I}=\log \frac{1}{T}$

$\therefore \quad \propto=2.303 \frac{\mathrm{A}}{\mathrm{X}}$

حيث أن T تمل الفانية

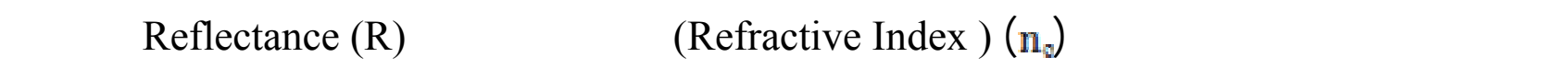

$R=\left[\frac{\left(n_{0}-1\right)^{2}+k_{0}}{\left(n_{0}+1\right)^{2}+k_{0}}\right]$

( د داؤد، 2000).

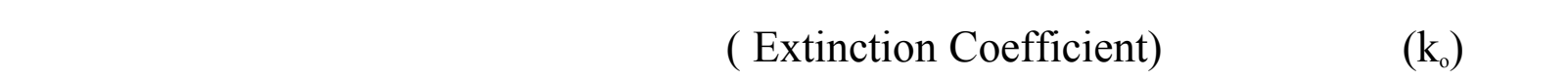

$\mathrm{n}_{\mathrm{s}}=\left[\left(\frac{1+\mathrm{R}}{1-\mathrm{R}}\right)^{2}-\left(\mathrm{k}_{\mathrm{s}}{ }^{2}+1\right)\right]^{1 / 2}+\left(\frac{1+\mathrm{R}}{1-\mathrm{R}}\right)$

منطاقة فوتونات المعاع للسقط عليها.

مه عسلب الانعكلسية من المعادلة الآتية (Nadeem and Ahmed, 2000).

$\mathrm{R}=1-\mathrm{A}-\mathrm{T}$

وتعرف الانعكلسية بأنها النبة بين شدة الشعاع المنعكس إلىشة للشعاع اللسقط عند الحد الفاصل بين ونطين. ويمكن الحصول على معلمل الخمود من العلاقة (Ugwa and Onah, 2007):

$\mathrm{k}_{\mathrm{s}}=\frac{\alpha \lambda}{4 \pi}$

حيث أن $\lambda$ الطول الموجي

أما ثابت العزل الكهربائي المعدد (ع) ( Dielectric Constant ) فيعرف على أنه الإنظطلب الحاصل لشحنات مادة الوس ط نتيجة الم تمصاص طاقة الشعاع اللساقط من قل شحنات الوسط ويطى بالمعادلة .(Nadeem and Ahmed, 2000 )

$\varepsilon=\left(\mathrm{n}_{\mathrm{s}}-\mathrm{ik}\right)^{2}$

$\varepsilon=\varepsilon_{1}-\varepsilon_{2}$ 
حيث أن ع ع ع الأجزاء الحقيقية والخيالية على التوالي لثابت العزل الكهربائي:

$$
\begin{aligned}
\therefore \varepsilon_{1} & =n_{0}^{2}-k_{0}^{2} \\
\varepsilon_{2} & =2 n_{0} k_{g}
\end{aligned}
$$

وقم حسلب ثابت العزل الكهربائي الحقيقي 10عن المعادلة رقم (10)

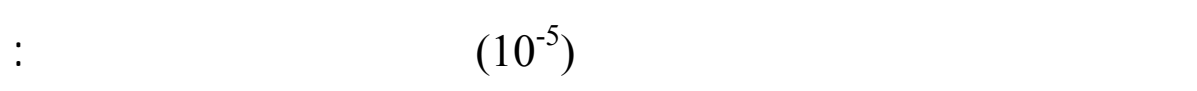

$$
\varepsilon_{1}=n_{0}^{2}
$$

تعرف التوصيلية البصرية الضوئية (o)( Optical Conductivity) على أنها ميل المادة للتوصيل

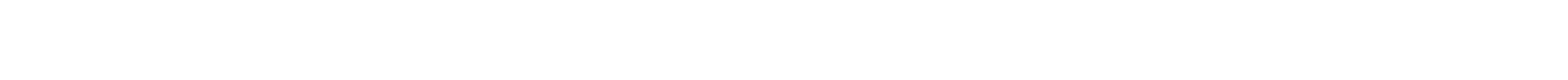
(Ezekoye and Okeke, 2006)

$$
\sigma=\frac{a n_{s} c}{4 \pi}
$$

حيث أن c تملسسرعة الضوء في الفراغ. ويمكن الحصول على القيمة النهائية لمعل جرعة الثٔشعة ف ـوق البفس جية UV ذاعل ذات الطول الموجي

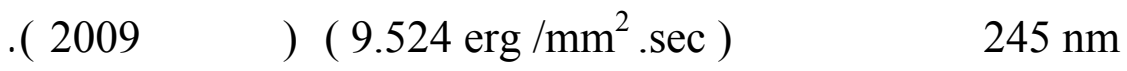
وأن الجرعة= معل الجرعة × زمن التشعبع

\section{الج لب المهـلي}

\section{المواد المستهماة وتحضير العينات المهاب}

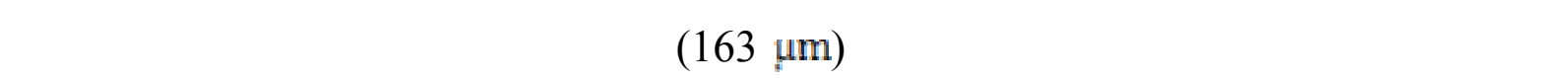
ولكل عينة بمسلحة ( 4 × 4 cm بما يتنلهب مع مسلحة الخلية المستخمة في جهاز التحليل الطيفي،

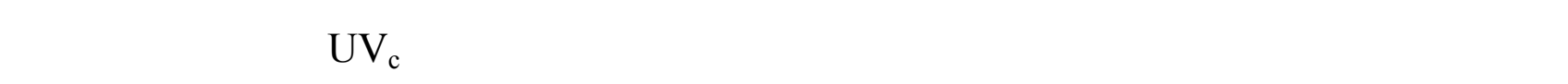

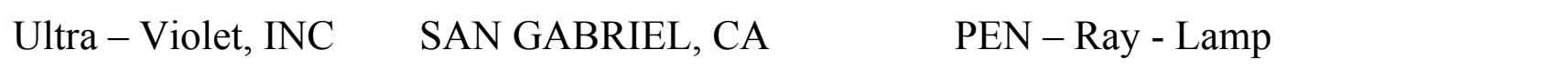

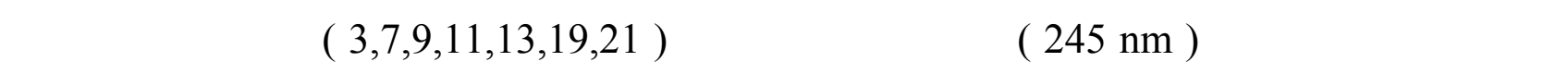


العينت على بعد 4 من المصدر المشع المذكور أعلاه، مّ تحليل العينت بعد عملية التشعبع بجهاز التحليل الطيفي.

\section{الفي ـ ـ ـلس ـ ـ ــ الع ـ ـ ملية}

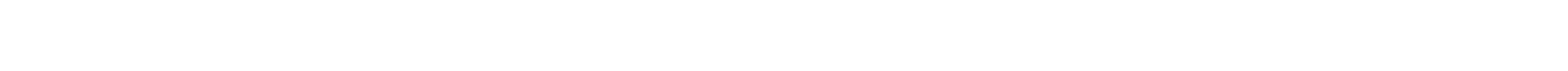

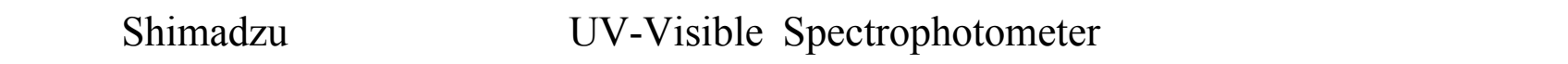
UV-210A ألموجي. ولأن توزيع مرلكز الامتصاص منظم خلال العينة لذا فان كمية الضوء الممتص من قبل الغثاء

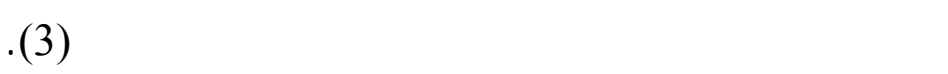

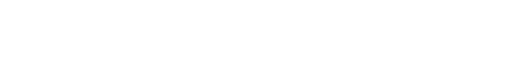

يوضح الثكل (1) العلاقة بين الامتصاصية للفلم الثبعاعي المستخدم في التثخيص الطبي والطٔول الموجية بلستخدلم الأشعة فوق البفسجية UV ولجرع تقفاوت بين (

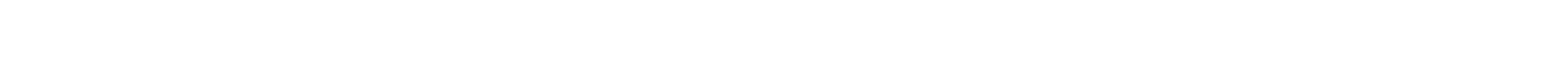

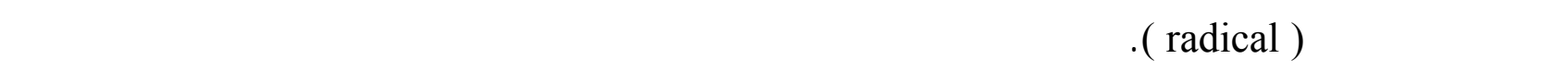

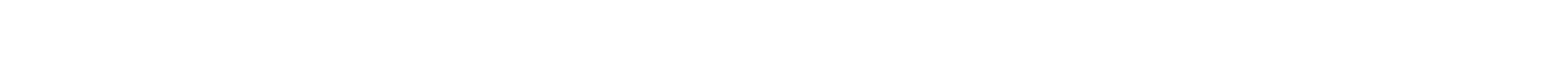
زيادة الامتصاصية للأشعة للساتطة لكثر من لمتصاص المادة غير المشعة، فكلما ازدادت كمية الجرعة

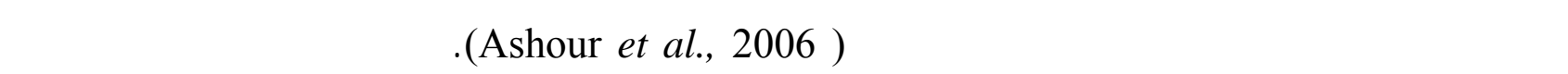
لمتصاصية ضوئية قع عند الطاول الموجي ( nm 342 ) لجمبع العينت المشععة. 


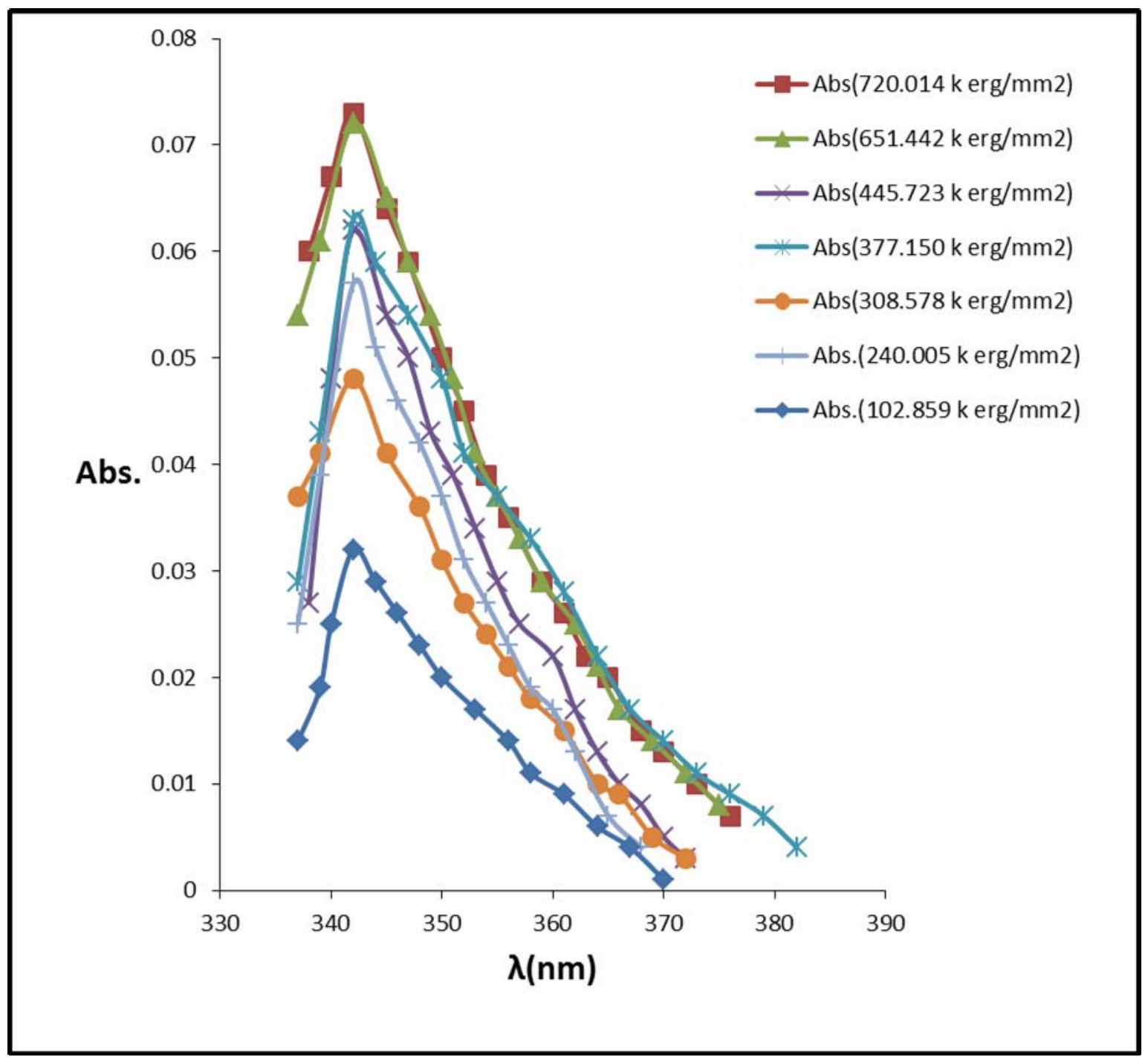

\section{اللثل 1 : يوضح اللفة بين المتصاصية الفوئية الفلم الفوتوغرالي والملطل الموبي ولجرعمفاوتة.}

للثكل (2) بييّن التغير الحاصل بن معلمل الخمود k والطول الموجي بلستخده UV

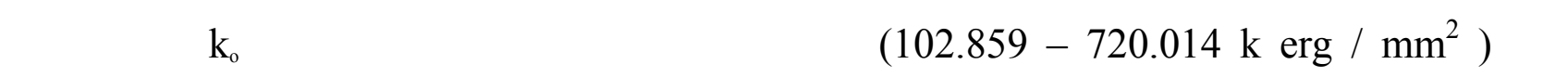

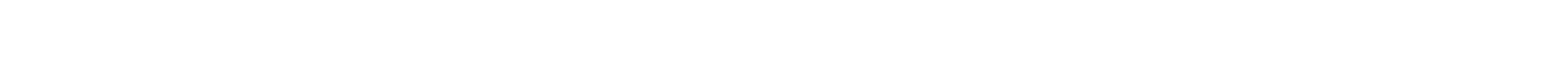

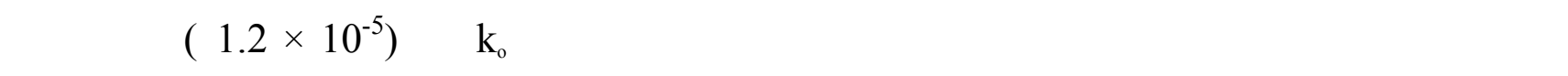

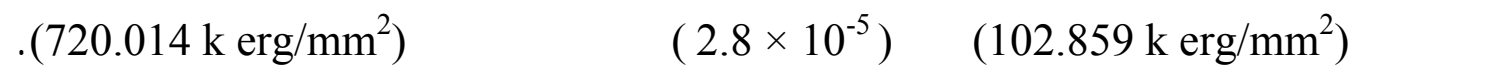




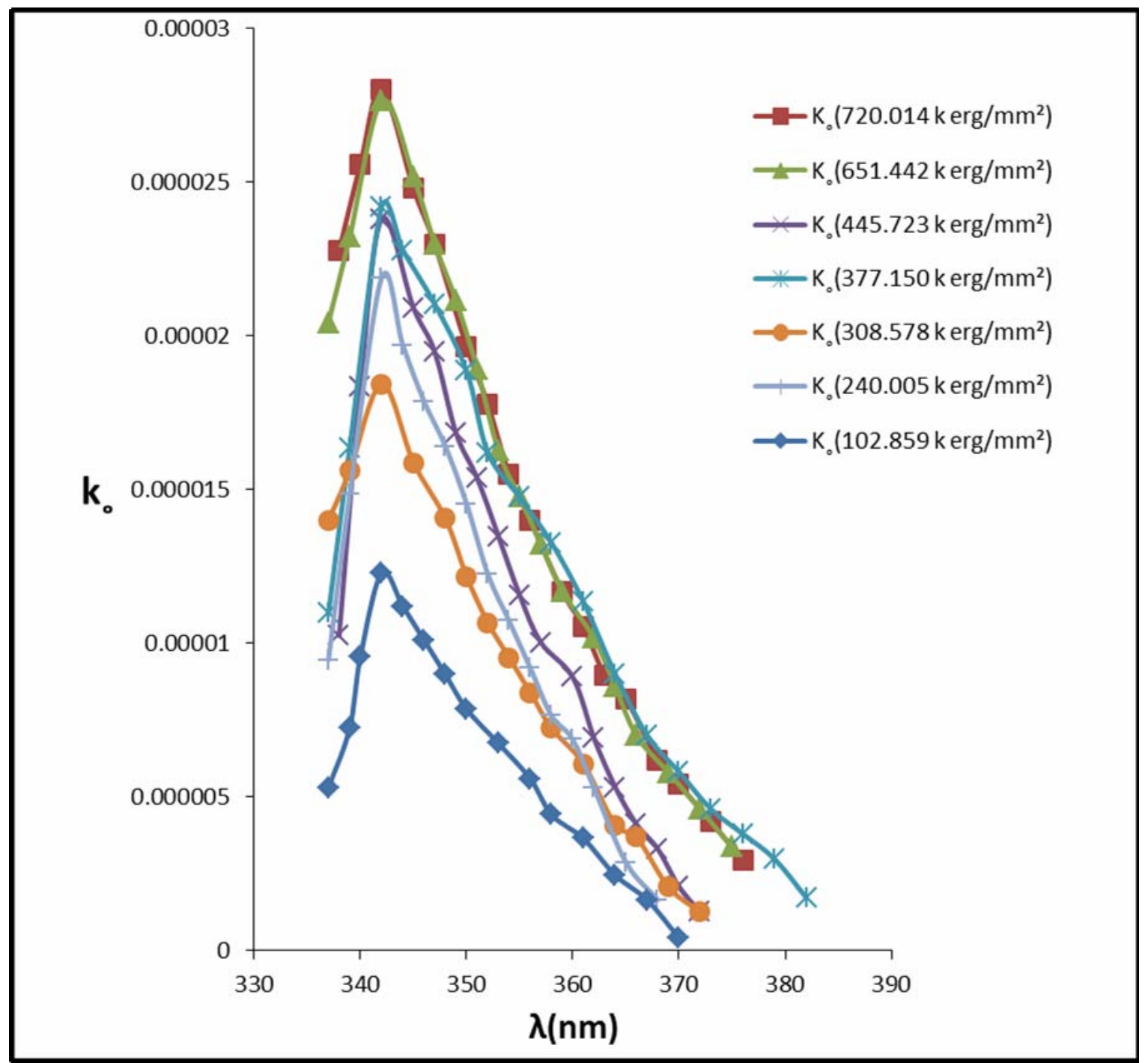

الشكل 2 : تخير معالم الخمود ko الفلم الفوتوغرالي مع اللال الموبي ولجرع مفاوتة.

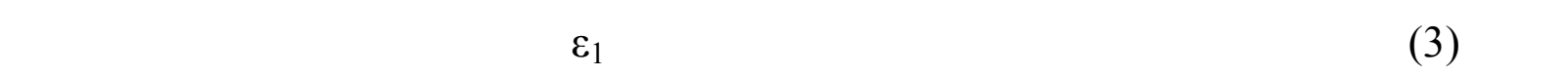

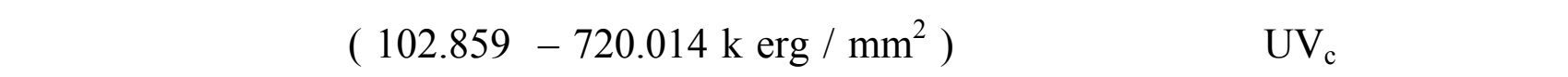

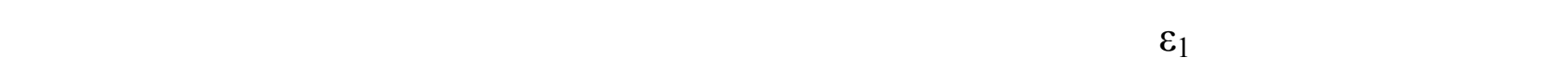

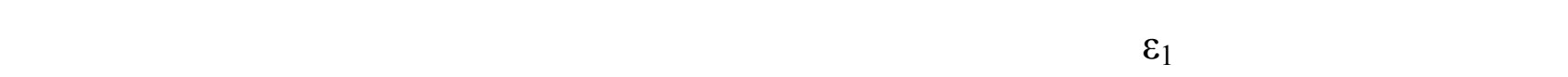

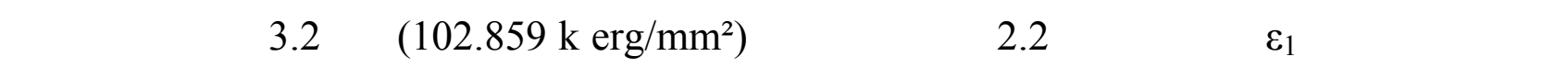
مقدارها (720.014 k erg/mm². 


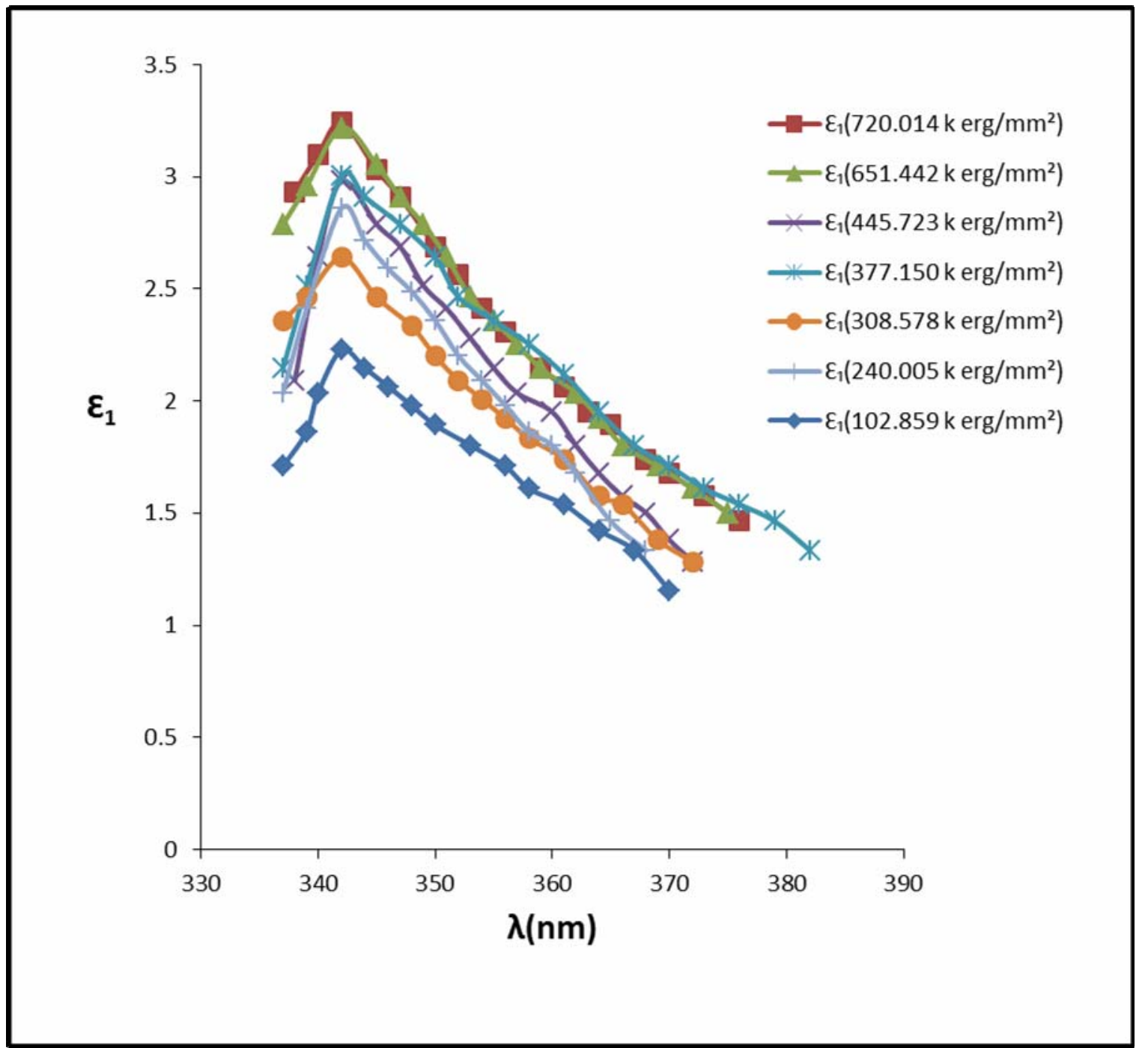

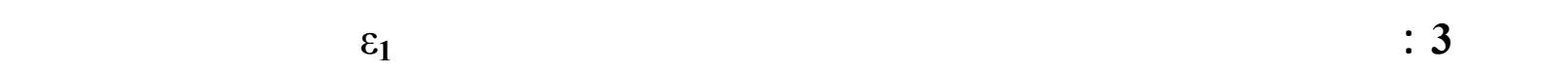 والملط الموجي ولجرع مفاوتة.}

للشكل (4) بيسين تغير الت -وصيلية البصرية الضوئية م مع الطول الموجي بلست خدلم الأث عة ف -وق

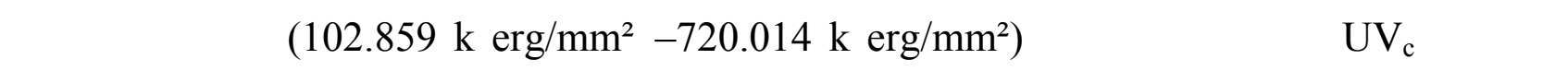

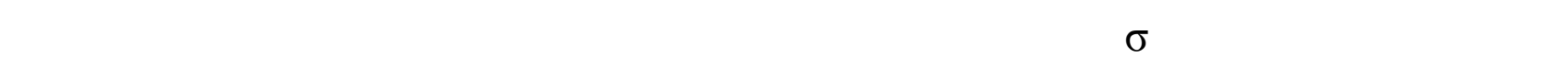
الشعاع الضوئي، فقد ازدادت قيمة التوصيلية البصرية الضوئية م من (102.85 (102.859 k erg/mm²) 


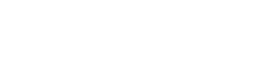

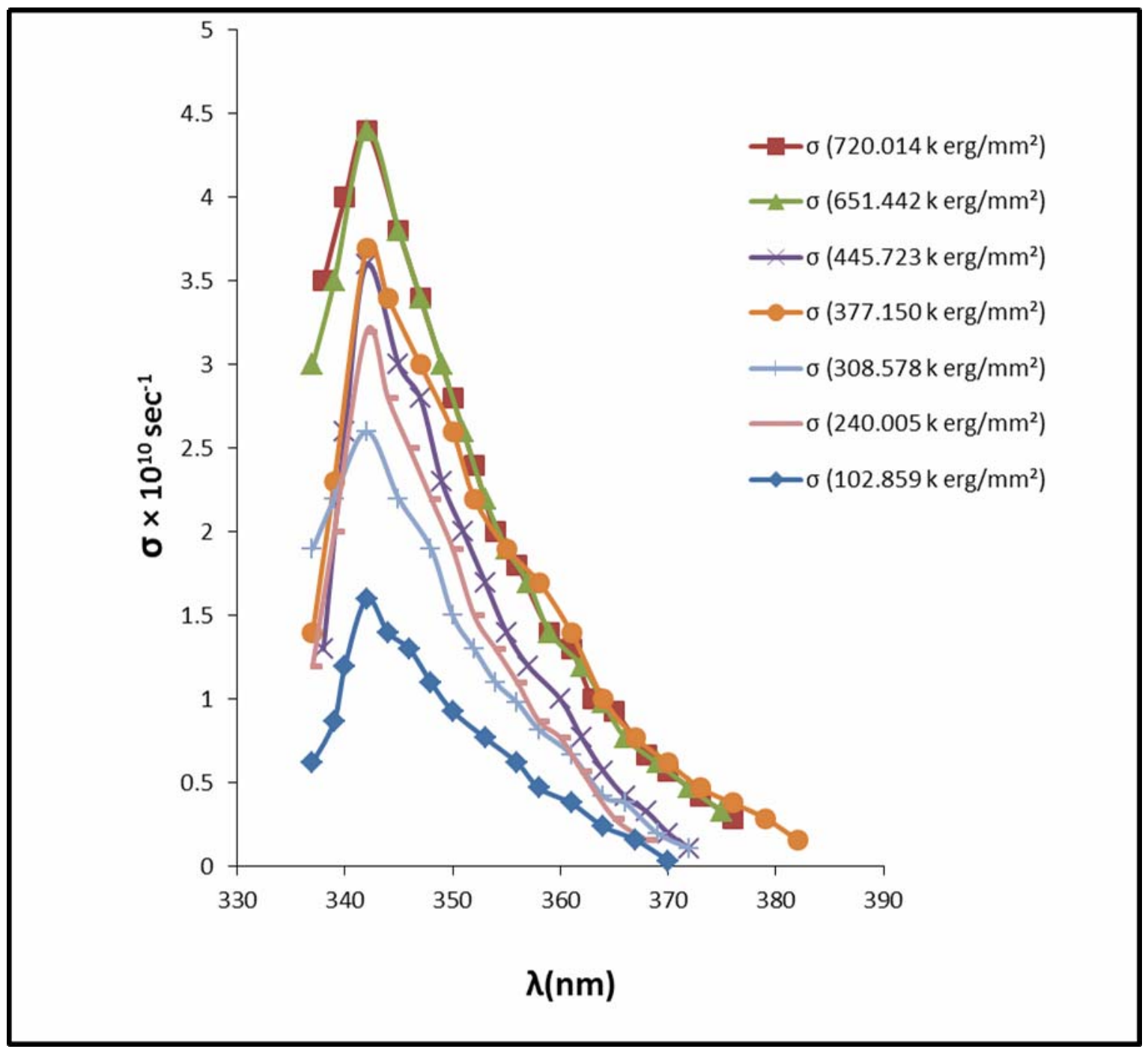

اللثل 4: تغير التوصيلية البصرية الضوئية ه الفلممع الللطل الموجي ولجرعمفاوتة.

يوضح الشكل( (5), (a,b,c,d ) العلاقة مابين قمم المتصاصية الضوئية .Abs وقمم معلمل الخمود

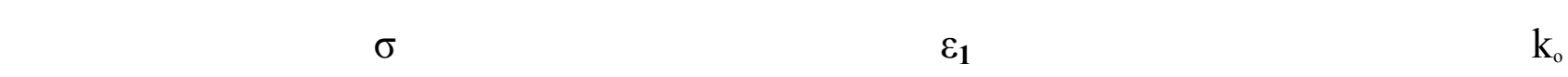
البفسجية UV على التوالي. 

إمكانية لستخدلم أفلام التشخيص الفوتوغرافي.

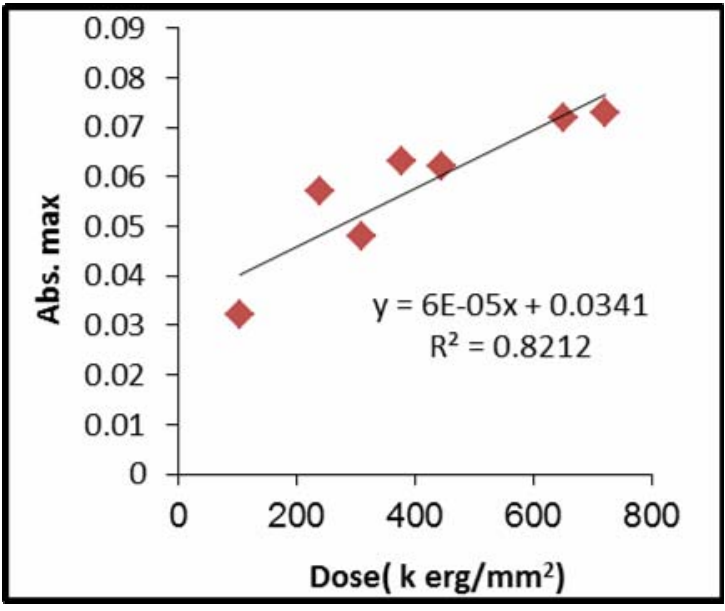

(a)

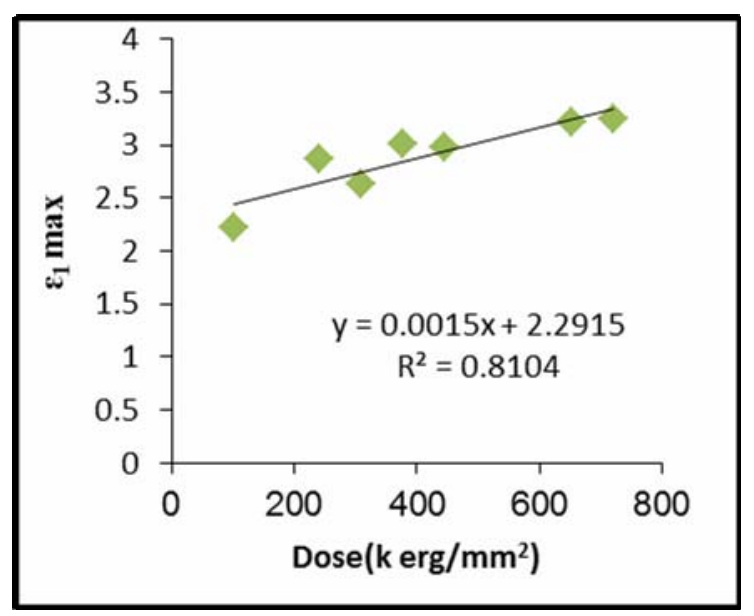

(c)

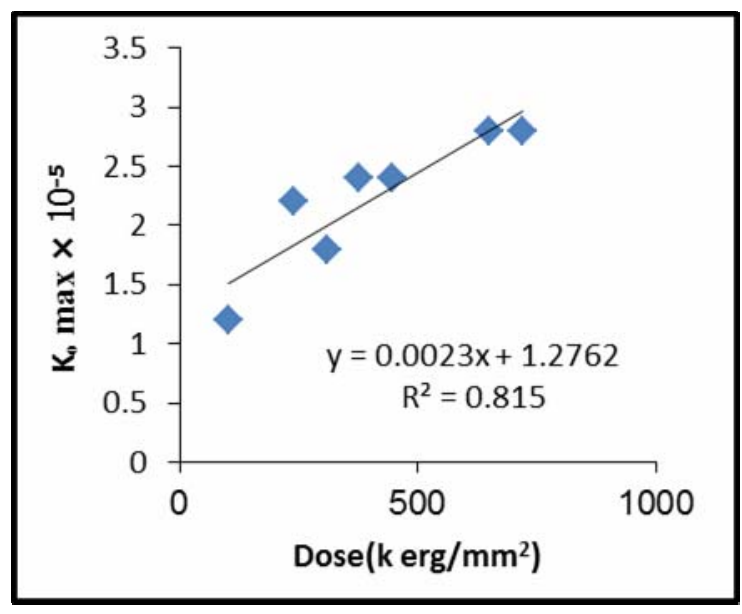

(b)

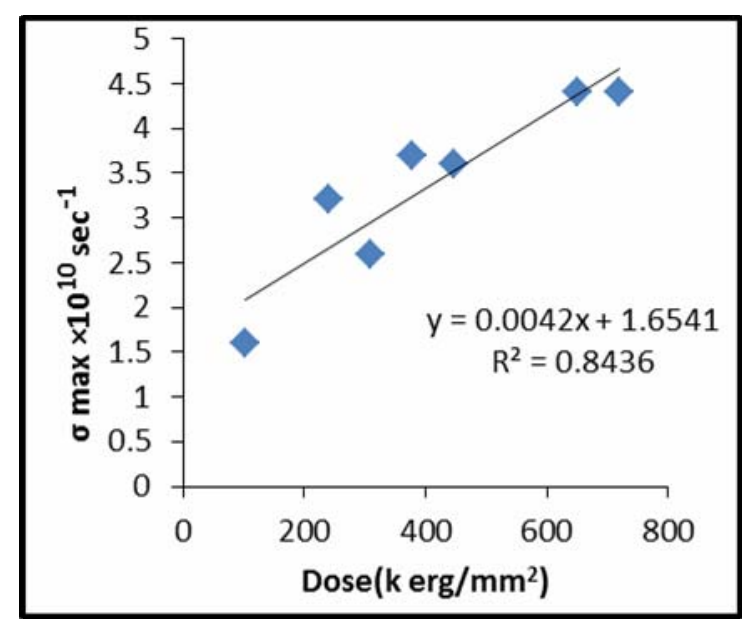

(d)

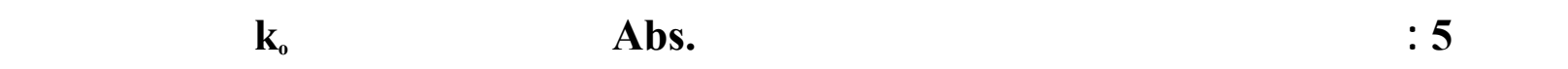

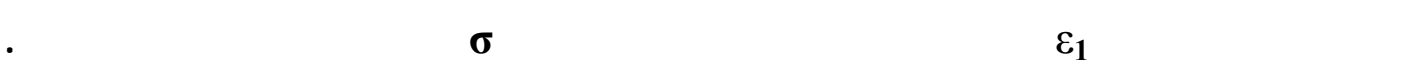

من خلل الشكل (5) مَ التوصل إلى معادلات خطية بملائمة (R²=0.8) تربط الجرعة الشعاعية مع الخواص أعلاه، والتي يمكن من خلالها توصيف الفلم الفوتوغرافي المستخه في هذا البحث مجراعَ للأشعة فوق البنفسجية UV وكما مبين أننه. بالنسة للشكل (a) المعادلة الغطية التي تربط الجرعة مع الامتصاصية الضوئية .Abs تكون كالآتي : Abs.max $=6 \times 10^{-5}$ Dose $\left(\mathrm{k} \frac{\mathrm{erg}}{\mathrm{mm}^{2}}\right)+0.0341$

وبالنبة للثكل (b) تكون المعادلة الخطية التي تربط الجرعة معمعلمل الخمود k كالآي : $\mathrm{k}_{\mathrm{s}} \max \times 10^{-5}=2.3 \times 10^{-3}$ Dose $\left(\mathrm{k} \frac{\mathrm{erg}}{\mathrm{mm}^{2}}\right)+1.2762$ 
وبالنسبة للثكل (c) المعادلة الخطية التي تربط الجرعة مع ثابت العزل الكهربائي الحقيقي 1ع تكون $\varepsilon_{1} \max =1.5 \times 10^{-3}$ Dose $\left(\mathrm{k} \frac{\mathrm{erg}}{\mathrm{mm}^{2}}\right)+2.2915$

أما بالنبة للشكل (d) تكون المعادلة الطية التي تربط الجرعة مع التوصيلية البصرية الضوئية كالآتي : $\sigma \max \times 10^{10}\left(\mathrm{sec}^{-1}\right)=4.2 \times 10^{-3}$ Dose $\left(\mathrm{k} \frac{\mathrm{erg}}{\mathrm{mm}^{2}}\right)+1.6541$

\section{لالستنتلج لت}

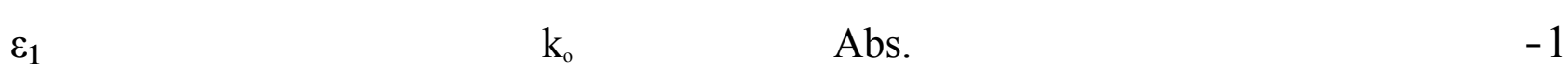

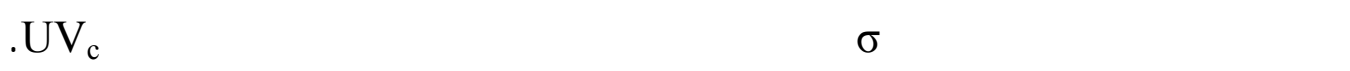

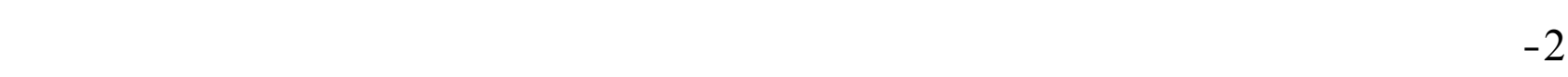

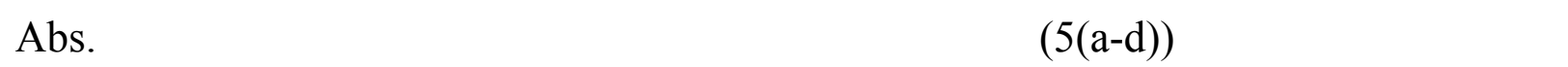
ومعلط الخمود k وثلابت العزل الكهربائي الحقيقي 1ع والتوصيلية البصرية م على التوالي.

\section{المصادر الد ـرية}

الجبوري، مسين علي لحمد (1984). تأثير الأشعة المؤينة وفوق البفسجية على نسبة نضوح الهيموكلوبين

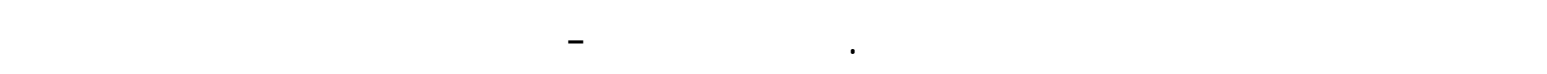

بغداد، العراق.

الجميلي، فرلس محمد علي فتهي (2009). قيلس الجرعك للشعاعية الكهرومغنلطيسية بلستعمل كولثف

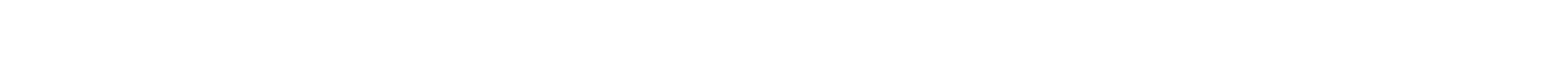

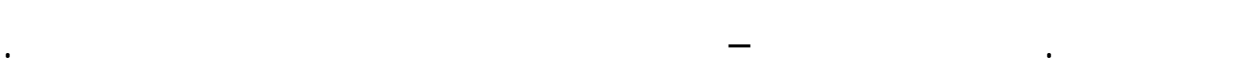

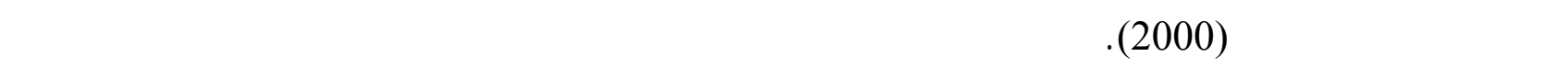
للأغشية الرقيقة SO . كسالة ملجستير - الجلمعة المستتصرية، بغداد، العراق.

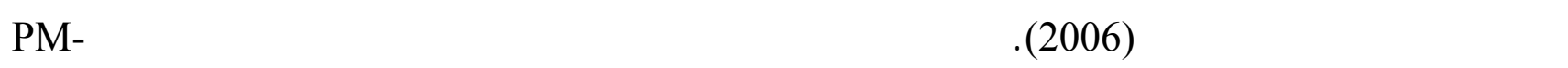

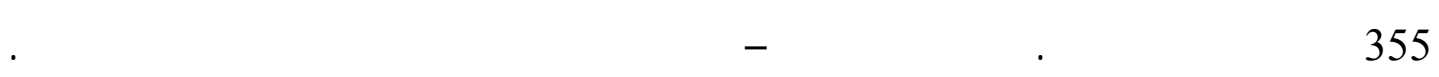

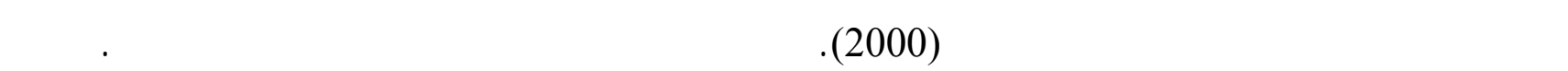
التربية والعلم. (43)، 65-55. 


\section{المصادر الأجنبية}

Akber, R. A.; Nadeem, K. H.; Majid, C. A.; Hussain, A.; Zaman, N.; Chaudhary, M. A.; Kham, H. A. (1980). Studies of structural changes produced by high doses of $\gamma-$ rays in some plastic track detectors. Nucl. Instr. and Meth., In Phys. Research, 173, 217- 221.

Ashour, A. H.; Saad, H. M.; Ibrahim, M. M. (2006). Electrical conductivity for irradiated, grafted polyethylene and grafted polyethylene with metal complex. Egypt. J. Solids, 29(2), 351-360

Deshmukh, S. H.; Burghate, D. K.; Shilaskar, S. N.; Chaudhari, G. N.; Deshmukh, P. T. (2008). Optical properties of polyaniline doped PVC - PMMA thin films. Indian $J$. Pure and Appl. Phys., 46, $344-348$.

Ezekoye, B. A.; Okeke, C. E. (2006). Optical properties in $\mathrm{PbHgS}$ ternary thin films deposited by solution growth method. The Pacific J. Sci. Technol., University of Nigeria, Nsukka, 7, (2).

Fluka, (2007/2008). "Laboratory Chemicals and Analytical Reagents". Fluka, chemic AG, RdH. 1652 p.

Meredith, W. J.; Massey, J. B. (1977). "Fundamental Physics of Radiographic". John Wright, Bristol. 5 p.

Nadeem, M. Y.; Ahmed, W. (2000). Optical properties of ZnS thin films. Turk J. Phys., 24, $651-659$.

Nagpal, J. S. (2000). Ultraviolet radiation dosimetry- perspectives, problems and parameters. Radiat. Protect, 24(5), 83-88.

Shweikani, R.; Raja, G.; Sawaf, A. A. (2002). The possibility of usining plastic detector CR-39 as UV- dosimeter. Radiat. Measurements, 35 (1350- 44870) 281-285.

Singh, S.; Prasher, S. (2004). UV-vis. Spectroscopic and etching studies of IR exposed CR39 plastic track detector. Nucl. Inst. And Method in Phys. Research B 215, 169-173.

Srivastava, A.; Virk, H. S. (2000). Modification of optical and structural response of CR-39 polymer by $50 \mathrm{MeV}$ Lithium ion irradiation. Conference Nuclear Track in Solids, Nuclear Society of Slovenia.

Stuart, C. W.; Michael, J. P. (2000). "Principles and Interpretation". 4th ed., John Scbrefer, United States St. Louis London Philadelphia Sydney Toronto, 2000.

Tanu, S.; Sanjeev, A.; Shyam, K.; Mittal, V. K.; Kalsi, P. C.; Manchanda, V. K. (2007). Effect of gamma irradiation on the optical properties of CR-39 polymer. $J$. Materials Sci., 42 (4),1127-1130.

Ugwa, E. I.; Onah, D. U. (2007). Optical characteristics of chemical bath deposited CdS in film characteristics within UV, visible and NIR radiation. The Pacific J. Sci. and Technol., 8, (1).

Wong, C. F.; Fleming, R. A.; Carter, S. J.; Ring, I. T.; Vishrakar, M. D. (1992). Measurement of human exposure to ultraviolet-B solar radiation using CR-39 dosimeter. Health Phys., 63(4), 457-461. 